\title{
Percepciones de Alumnos del Grado en Maestro en Educación Primaria con Mención en Educación Física sobre la Adquisición de Competencias
}

\author{
Students' Perceptions of the Acquisition of Competences in a Degree Program \\ in Primary Teaching with Concentration in Physical Education
}

\author{
Carlos Gutiérrez ${ }^{a}$, David Hortigüela ${ }^{b}$,Zara Peral ${ }^{c}$, Angel Pérez Pueyo ${ }^{d}$ \\ Universidad de León \\ Correo electrónico: cgutg@unileon.es \\ Universidad de Burgos \\ Correo electrónico: dhortiguela@ubu.es \\ Universidad de León \\ Correo electrónico: zaraperal@hotmail.com \\ Universidad de León \\ Correo electrónico: angel.perez.pueyo@unileon.es
}

\begin{abstract}
RESUMEN
El objetivo de este trabajo fue conocer y comparar la percepción sobre competencias adquiridas que tienen los estudiantes del Grado en Magisterio con mención en Educación Física al finalizar sus estudios. Se realizó un estudio descriptivo de poblaciones de tipo transversal, en el que 699 estudiantes de diez universidades públicas españolas cumplimentaron el Cuestionario sobre competencias docentes en la formación inicial del profesorado de Educación Física (CCDFIPEF). Globalmente, los estudiantes consideraron que su formación inicial como maestros había desarrollado sus competencias personales generales, docentes genéricas y específicas de Educación Física de forma similar, en un punto intermedio entre "medianamente" y "mucho". Específicamente, existieron dos competencias que los estudiantes manifestaron haber desarrollado mucho, mientras que cinco obtuvieron una baja valoración y las 41 restantes se situaron en posiciones intermedias. Esta investigación también concluye que existen similitudes y diferencias de percepción atendiendo a la universidad donde se realizaron los estudios.

Palabras claves: formación inicial del profesorado, maestros, educación, estudiantes.
\end{abstract}

\begin{abstract}
The objective of this study was to determine and compare the perceptions of students in a primary education physical education concentration degree program regarding the competences they acquired by the end of their studies. A cross-sectional, descriptive population study was designed in which 699 students from ten Spanish public universities completed a questionnaire on teaching competences in physical education teacher education (CCDFIPEF). The overall results showed that the students considered that their initial training as teachers had developed their generic personal skills, generic teaching skills, and specific physical education teaching skills in a similar way, with responses for most skills falling between "moderately" and "considerably." Additionally, there were two competences that students expressed they had developed more than considerably, while five obtained scores lower than moderately. The remaining 41 competences received intermediate scores. This research also concludes that there were both similarities and differences in students' perceptions of competences based on which university they attended.
\end{abstract}

Keywords: initial teacher training, teachers, education, students. 
Estudios Pedagógicos XLIV, N $^{\circ}$ 2: 223-239, 2018

PERCEPCIONES DE ALUMNOS DEL GRADO EN MAESTRO EN EDUCACIÓN PRIMARIA CON MENCIÓN EN EDUCACIÓN FÍSICA SOBRE LA ADQUISICIÓN DE COMPETENCIAS

\section{INTRODUCCIÓN}

En el año 2013 se titularon en España las primeras promociones de graduados en maestro en Educación Primaria. Los nuevos egresados se enfrentaban a un panorama laboral incierto, derivado de la fuerte crisis económica que sufría el país desde 2008, y con la que habían tenido que convivir durante toda su trayectoria universitaria. En el periodo 20092013, la inversión global en educación se recortó en torno a un 16,6\%, cifras inéditas en la historia reciente del país (Martínez, 2016), y los años sucesivos tampoco mejoraron sustancialmente esta realidad (Viaña, 2017). Las consecuencias de esta crisis se han materializado en numerosos ámbitos de la esfera educativa. Entre otros, disminución de becas y ayudas, aumento de las exigencias para su concesión, incremento de las ratios profesor-alumno, disminución de apoyos para alumnos con dificultades de aprendizaje y necesidades educativas específicas, falta de renovación y nueva construcción de centros escolares, pérdida de profesorado, falta de renovación de las plantillas, con su consiguiente envejecimiento, aumento del empleo interino, congelación e incluso reducción de sueldos del profesorado, etc. (Díaz \& Hervella, 2017; Martínez, 2016).

La universidad, obviamente, no ha sido ajena a esta situación. La crisis impuso importantes restricciones de financiación a las universidades que no solo han afectado a la realidad de la docencia, investigación, transferencia y gestión que desarrolla el personal universitario, sino también al alumnado. En pocos años se elevaron sustancialmente las tasas para cursar estudios universitarios y se endurecieron los requisitos para la concesión de becas. Estos factores se han apuntado como parcialmente responsables de los casi 100.000 alumnos que perdieron las universidades españolas entre los cursos 2011-2012 y 2014-2015 (Parellada, 2015). Según se señalaba en el epílogo del resumen ejecutivo del informe anteriormente citado

El impacto de la crisis en la universidad española ha sido, y es, de una importancia muy notable. No existen precedentes en estas últimas décadas de un impacto de estas características tan prolongado en el tiempo. Podremos seguramente hablar muy pronto de la década perdida del sistema universitario español. (Parellada, 2015, p. 25).

Uno de los aspectos lamentables de esta situación fue su coincidencia con procesos fundamentales para la implantación definitiva de las nuevas titulaciones adaptadas al Espacio Europeo de Educación Superior. Esta implantación exigía, al menos sobre el papel, un esfuerzo importante al profesorado en ámbitos como la renovación de las metodologías de enseñanza y de evaluación o la utilización de nuevas tecnologías, que se ha percibido como poco valorado institucionalmente (Pozo \& Bretones, 2015). Con todo, son muchos los profesores que han realizado este esfuerzo, lo que ha permitido que actualmente en España sean conocidos y aplicados muchos términos ligados a la renovación de la enseñanza y al desarrollo de competencias. Sirvan como ejemplo buenas prácticas, actividades auténticas, evaluación formativa, evaluación para el aprendizaje, evaluación auténtica, TIC, TAC, etc. (Brown \& Pickford, 2013; López-Pastor \& Pérez-Pueyo, 2017; Lozano, 2011; Vallejo \& Molina, 2014).

El presente estudio se encuadra en este panorama reciente de transformaciones, dificultades y renovación del sistema universitario español, en una línea de trabajo relacionada con las percepciones de competencia que tienen los alumnos del Grado en 
Maestro de Educación Primaria con Mención en Educación Física. En este sentido, la percepción de competencia ha sido estudiada en otras investigaciones relacionadas con la formación del profesorado (Cañabate et al., 2014; Díez et al., 2009; Gabarda et al., 2017; Gallego \& Rodríguez, 2014, 2015; Gutiérrez-García et al., 2011; Hortigüela, PérezPueyo \& Fernández-Río, 2016b; Iglesias, 2009; Izuzquiza, Echeita \& Simón, 2015; Romero \& Oliveira, 2011; Romero et al., 2017), y es un aspecto relevante por cuanto muestra cuánto de competente se siente el futuro profesorado para ejercer su labor. Se trata de una perspectiva parcial que debe complementarse con otras (percepción del profesorado, egresados, empleadores, futuros alumnos y otros tipos de evaluaciones) y que puede orientarse a la mejora de la formación inicial del profesorado, de ahí su valor. Concretamente, los objetivos de este estudio fueron dos. En primer lugar, conocer la percepción de competencias adquiridas que tienen los alumnos del Grado en Maestro en Educación Primaria con Mención en Educación Física de diversos centros de formación de universidades españolas al finalizar sus estudios; y, en segundo lugar, comparar dicha percepción entre los alumnos de los diferentes centros de formación.

\section{MÉTODO}

Se diseñó un estudio descriptivo de poblaciones mediante encuestas de tipo transversal (Montero \& León, 2007), ya que se consideró el más adecuado para atender los objetivos descriptivo-comparativos de la investigación, ligados a las percepciones de los encuestados sobre sus propias competencias (i.e., el estudio no analizó las competencias reales de los estudiantes, sino sus percepciones sobre las mismas). Asimismo, la selección de la encuesta como técnica de investigación permite alcanzar de forma eficaz muestras amplias de participantes (Sierra, 1994).

\subsection{MUESTRA}

Un total de 699 alumnos de último curso del Grado en Magisterio con Mención en Educación Física de diez universidades públicas españolas participaron en este estudio. La distribución de la muestra en función del género fue ligeramente mayor en mujeres $(n=$ $366 ; 52,4 \%)$ que en hombres $(n=333 ; 47,6 \%)$, con una primacía del rango de edad de 22 23 años $(n=318 ; 45,5 \%)$. Un 72,5\% del alumnado $(n=507)$ accedieron a la universidad a través de bachillerato, el 23,5\% $(n=164)$ tras realizar el ciclo de Formación Profesional de Grado Superior de Técnico de Animación de Actividades Físicas y Deportivas, y el 0,9\% $(n=6)$ mediante el acceso a la universidad para mayores de 25 años. Para otro 1,9\% $(n=$ 13) era su segunda titulación y un $0,6 \%(n=4)$ estaban realizando la adaptación al Grado desde la antigua Diplomatura en Magisterio.

\subsection{INSTRUMENTO}

Para la recogida de datos se utilizó un cuestionario específico denominado "Cuestionario sobre competencias docentes en la formación inicial del profesorado de Educación Física (CCDFIPEF)". Este cuestionario dispone de tres versiones muy similares en función de que sus destinatarios sean alumnos, profesores universitarios o egresados. La versión 
para alumnos del CCDFIPEF (CCDFIPEF-A), utilizada en la presente investigación, se compone de dos partes fundamentales. La primera parte se orienta al conocimiento de aspectos relativos a competencias docentes, metodologías, evaluación, calificación y satisfacción respecto a los estudios cursados. Consta de nueve preguntas en las que se integran un total de 76 ítems que en su gran mayoría (excepto las preguntas ocho y nueve) se responden a través de una escala tipo Likert de cinco niveles que responden a (0) muy poco o nada, (1) poco, (2) medianamente, (3) mucho, y (4) muchísimo. La segunda parte del cuestionario se orienta a recoger datos para la caracterización de la muestra (estudios cursados, sexo, edad y acceso a la universidad).

Concretamente, para este estudio se han utilizado las dos primeras preguntas del cuestionario (46 ítems en total), centradas en el desarrollo de competencias docentes. Éstas se han agrupado en tres grandes dimensiones: (1) Competencias genéricas o personales, comunes a cualquier graduado, con 12 ítems; (2) Competencias docentes genéricas, es decir comunes a cualquier docente independientemente de su especialidad, con 12 ítems; y (3) Competencias docentes específicas de Educación Física, con 22 ítems. La cuestión inicial en ambas preguntas es "Teniendo en cuenta todas las asignaturas cursadas en la carrera ¿En qué grado crees que te han ayudado a desarrollar las siguientes competencias docentes?".

La elaboración del cuestionario se realizó en el seno de la Red de Evaluación Formativa y Compartida en Educación Superior (REFYCES), y siguió tres fases: (1) En primer lugar, se recopiló un conjunto inicial y amplio de ítems a partir del análisis de estudios e informes relacionados con el tema (ANECA, 2005a, 2005b; Carreras \& Perrenoud, 2005; Galvis, 2007; Gutiérrez-García et al., 2011; Perrenoud, 2004; Romero et al., 2011); (2) Este conjunto de preguntas fue evaluado por diez expertos en Didáctica de la Expresión Corporal y de la Educación Física, a los que se pidió que seleccionasen aquellos ítems más pertinentes por su relevancia y claridad; (3) Se realizaron los pre-test con alumnos de características similares al objeto de estudio, para asegurar la comprensión de los ítems y del cuestionario en su conjunto. A partir de sus aportaciones, se fijó la versión final de la escala. La fiabilidad de las tres dimensiones analizadas, calculada mediante el Alfa de Cronbach, fue muy alta (DeVellis, 2003), oscilando entre 0,879 y 0,921. El CCDFIPEF ya ha sido utilizado en otros estudios de la REFYCES (Lorente-Catalán et al., 2017; RomeroMartín et al., 2017).

\subsection{VARIABLES}

Para atender al primero de los objetivos del estudio, se tuvieron en cuenta las siguientes variables: (a) competencias estudiadas (de forma individual), (2) dimensiones de competencias (genéricas o personales, docentes genéricas y específicas de Educación Física), y (3) perfil competencial total. Para atender al segundo de los objetivos, relativo a la comparación entre centros de formación, se tomó como variable dependiente la pertenencia de los alumnos a los diversos centros, y como variables independientes las dimensiones de competencias (genéricas o personales, docentes genéricas y específicas de Educación Física), y el perfil competencial total. No se consideraron como variables independientes las competencias concretas debido al excesivo sobredimensionamiento que habría tenido el estudio y por no considerarlo muy importante para evidenciar, desde una perspectiva general, las diferencias que podrían existir entre los centros de formación. 


\subsection{PROCEDIMIENTO}

Con el objeto de comprobar la autopercepción de competencia de los alumnos que finalizan el Grado en Magisterio con Mención en Educación Física, profesores e investigadores de la REFYCES administraron el cuestionario por escrito a los grupos de alumnos una vez en una de las clases finales del último curso del Grado en Magisterio (mención en Educación física) del año 2014-2015. El cuestionario fue autocumplimentado por todos aquellos alumnos que accedieron a participar en el estudio. Previamente, estos alumnos fueron informados de la naturaleza del estudio y de sus objetivos, su carácter voluntario y anónimo, y se les rogó la máxima sinceridad en sus respuestas. El tiempo medio dedicado a cumplimentar la totalidad del cuestionario fue de diez minutos.

\subsection{ANÁLISIS DE DATOS}

Los datos de los cuestionarios fueron analizados con el programa IBM SPSS Statistics, versión 22.0. Se utilizaron estadísticos descriptivos (media y desviación estándar) para calcular las puntuaciones de las competencias y dimensiones de competencias. Por otro lado, se utilizó una estadística inferencial (análisis de varianza con pruebas post hoc de Bonferroni) para analizar la existencia de diferencias entre centros de formación, una vez analizada y asumida la normalidad de las variables. El nivel de significación se fijó en $p<0,05$.

\section{RESULTADOS}

Las puntaciones medias para la dimensión competencias personales generales fueron 29,94 \pm 7,15 (62,4\% del máximo teórico, 48 puntos), 26,67 \pm 7,72 para la dimensión competencias docentes genéricas (55,6\% del máximo teórico, 48 puntos), 52,98 \pm 11,70 para la dimensión competencias específicas de Educación Física (60,2\% del máximo teórico, 88 puntos), y 109,60 $\pm 23,43$ para el total de competencias $(59,6 \%$ del máximo teórico, 184 puntos).

Considerando individualmente cada una de las competencias, la Tabla 1 muestra sus puntuaciones medias en cada dimensión y orden decreciente.

Tabla 1. Media y desviación típica de la percepción de competencias

\begin{tabular}{|l|c|c|}
\hline Competencias & $\mathrm{M}$ & $\mathrm{DE}$ \\
\hline Competencias personales generales & 29,94 & 7,15 \\
\hline (1) Trabajo en equipo & 3,27 & 0,77 \\
\hline$(2)$ Habilidades en las relaciones interpersonales & 2,85 & 0,89 \\
\hline (3) Aprendizaje autónomo & 2,81 & 0,84 \\
\hline$(4)$ Creatividad & 2,68 & 0,99 \\
\hline (5) Razonamiento crítico & 2,68 & 0,86 \\
\hline
\end{tabular}


Estudios Pedagógicos XLIV, $\mathrm{N}^{\circ}$ 2: 223-239, 2018

PERCEPCIONES DE ALUMNOS DEL GRADO EN MAESTRO EN EDUCACIÓN PRIMARIA CON MENCIÓN EN EDUCACIÓN FÍSICA SOBRE LA ADQUISICIÓN DE COMPETENCIAS

\begin{tabular}{|c|c|c|c|}
\hline (6) & Adaptación a situaciones nuevas & 2,60 & 0,90 \\
\hline (7) & Compromiso ético & 2,57 & 0,94 \\
\hline (8) & Capacidad de organización y planificación & 2,54 & 0,83 \\
\hline (9) & Comunicación oral y escrita en lengua nativa & 2,51 & 0,97 \\
\hline$(10)$ & Capacidad de análisis y síntesis & 2,34 & 0,90 \\
\hline$(11)$ & Conocimientos de informática relativos al ámbito de estudio & 1,78 & 1,11 \\
\hline$(12)$ & Conocimiento de una lengua extranjera & 1,37 & 1,14 \\
\hline Compe & tencias docentes genéricas & 26,67 & 7,72 \\
\hline (1) & Organizar y animar situaciones de aprendizaje & 2,47 & 0,79 \\
\hline (2) & Trabajar en equipo con otros docentes & 2,46 & 1,07 \\
\hline (3) & Implicar al alumnado en su aprendizaje y en la vida del centro & 2,39 & 0,87 \\
\hline (4) & Gestionar la progresión de los aprendizajes & 2,35 & 0,78 \\
\hline (5) & Organizar la propia formación continua & 2,29 & 0,98 \\
\hline$(6)$ & Utilizar las tecnologías de la información y la comunicación & 2,26 & 1,09 \\
\hline (7) & Conocer características de la organización de los centros educativos & 2,18 & 0,91 \\
\hline (8) & Elaborar y poner en práctica estrategias de atención a la diversidad & 2,18 & 0,95 \\
\hline (9) & Elaborar propuestas de cambio de la realidad educativa & 2,16 & 0,92 \\
\hline$(10)$ & Afrontar los deberes y los dilemas éticos de la profesión & 2,13 & 0,97 \\
\hline (11) & Informar e implicar a las familias & 1,97 & 1,07 \\
\hline$(12)$ & Participar en la gestión del Centro & 1,86 & 1,04 \\
\hline Compet & tencias específicas de Educación Física & 52,98 & 11,70 \\
\hline & $\begin{array}{l}\text { Saber utilizar el juego como recurso didáctico y como contenido de } \\
\text { enseñanza }\end{array}$ & 3,09 & 0,79 \\
\hline (2) & $\begin{array}{l}\text { Conocer los fundamentos básicos de la iniciación deportiva escolar y } \\
\text { diseñar tareas específicas para utilizarlos en el ámbito de la enseñanza }\end{array}$ & 2,68 & 0,82 \\
\hline (3) & $\begin{array}{l}\text { Conocer los elementos y fundamentos de la expresión corporal y la } \\
\text { comunicación no verbal y su valor formativo y cultural }\end{array}$ & 2,62 & 0,88 \\
\hline (4) & Conocer el desarrollo psicomotor y su maduración evolutiva & 2,56 & 0,85 \\
\hline$(5)$ & $\begin{array}{l}\text { Analizar y comunicar, de manera crítica y fundamentada, el valor de la } \\
\text { actividad física y el deporte y sus posibilidades de contribuir al } \\
\text { desarrollo y bienestar de las personas }\end{array}$ & 2,51 & 0,79 \\
\hline (6) & $\begin{array}{l}\text { Saber aplicar los fundamentos (técnicas) de las actividades físicas en el } \\
\text { medio natural }\end{array}$ & 2,47 & 1,01 \\
\hline (7) & $\begin{array}{l}\text { Disponer estrategias de enseñanza que promuevan la adquisición de } \\
\text { hábitos de actividad física regular }\end{array}$ & 2,46 & 0,91 \\
\hline (8) & $\begin{array}{l}\text { Conocer las capacidades físicas y los factores que determinan su } \\
\text { evolución y saber aplicar sus fundamentos técnicos específicos }\end{array}$ & 2,45 & 0,81 \\
\hline (9) & Conocer y comprender los procesos evolutivos corporales y motrices & 2,44 & 0,82 \\
\hline
\end{tabular}




\begin{tabular}{|c|c|c|c|}
\hline (10) & $\begin{array}{l}\text { Tener capacidad de reflexión sobre el proceso de enseñanza- } \\
\text { aprendizaje, los diferentes tipos organizativos y las distintas } \\
\text { metodologías dentro de las clases de Educación Física }\end{array}$ & 2,43 & 0,82 \\
\hline (11) & $\begin{array}{l}\text { Conocer los fundamentos biológicos y fisiológicos del cuerpo humano } \\
\text { en relación con la actividad física }\end{array}$ & 2,39 & 0,90 \\
\hline (12) & $\begin{array}{l}\text { Diseñar, aplicar y analizar intervenciones didácticas en la asignatura de } \\
\text { Educación Física }\end{array}$ & 2,37 & 0,80 \\
\hline (13) & $\begin{array}{l}\text { Identificar y prevenir los riesgos que se derivan para la salud de la } \\
\text { práctica de actividades físicas inadecuadas }\end{array}$ & 2,36 & 0,93 \\
\hline (14) & $\begin{array}{l}\text { Diseñar, modificar y/o adaptar al contexto educativo situaciones } \\
\text { motrices orientadas al desarrollo y perfeccionamiento de las } \\
\text { habilidades motrices }\end{array}$ & 2,36 & 0,83 \\
\hline (15) & $\begin{array}{l}\text { Promover actividades complementarias relacionadas con la actividad } \\
\text { física y el deporte dentro y fuera del recinto educativo }\end{array}$ & 2,36 & 0,94 \\
\hline (16) & $\begin{array}{l}\text { Saber utilizar instrumentos de evaluación en la asignatura de Educación } \\
\text { Física }\end{array}$ & 2,35 & 0,91 \\
\hline (17) & $\begin{array}{l}\text { Conocer y promover las diferentes manifestaciones motrices que } \\
\text { forman parte de tu cultura tradicional }\end{array}$ & 2,34 & 0,84 \\
\hline (18) & $\begin{array}{l}\text { Diseñar, desarrollar y evaluar procesos de enseñanza-aprendizaje } \\
\text { relativos a competencia motriz, con atención a las características } \\
\text { individuales y contextuales de las personas }\end{array}$ & 2,30 & 0,83 \\
\hline (19) & $\begin{array}{l}\text { Disponer de estrategias de aplicación de los elementos de salud sobre } \\
\text { la higiene y alimentación en la práctica educativa }\end{array}$ & 2,29 & 1,00 \\
\hline (20) & $\begin{array}{l}\text { Diseñar, desarrollar y evaluar los procesos de enseñanza-aprendizaje } \\
\text { relativos a la actividad física y el deporte con atención a las } \\
\text { características individuales y contextuales de las personas }\end{array}$ & 2,27 & 0,78 \\
\hline (21) & Dar respuesta a la diversidad en las prácticas de Educación Física & 2,22 & 0,96 \\
\hline$(22)$ & $\begin{array}{l}\text { Elaborar y poner en práctica programas de Educación Física que } \\
\text { faciliten la inclusión efectiva de los alumnos con necesidades } \\
\text { educativas especiales }\end{array}$ & 1,88 & 0,94 \\
\hline
\end{tabular}

Como se puede observar, las competencias relacionadas con el trabajo en equipo y con la utilización del juego como recurso didáctico son las que obtuvieron una puntuación más elevada, por encima de tres sobre cuatro ("mucho-muchísimo"). En sentido contrario, es destacable que ninguna competencia obtuviese puntuaciones entre 0 y 1 ("nada-poco"), aunque cinco de ellas obtuvieron una puntuación inferior a 2 ("poco-medianamente"): las relacionados con la información a las familias, la facilitación de la inclusión efectiva a alumnos necesidades educativas especiales, la participación en gestión de centro, el conocimiento de informática aplicada al ámbito de estudio y el conocimiento lengua extranjera. El resto de las competencias analizadas alcanzaron una puntuación de entre 2 y 3 puntos ("medianamente-mucho"). 
Estudios Pedagógicos XLIV, $\mathrm{N}^{\circ}$ 2: 223-239, 2018

PERCEPCIONES DE ALUMNOS DEL GRADO EN MAESTRO EN EDUCACIÓN PRIMARIA CON MENCIÓN EN EDUCACIÓN FÍSICA SOBRE LA ADQUISICIÓN DE COMPETENCIAS

Por lo que se refiere a la comparación entre los centros de formación, la Tabla 2 muestra la media y desviación típica de las puntuaciones alcanzadas.

Tabla 2. Media y deviación típica de las dimensiones de competencias atendiendo al centro de realización de los estudios

\begin{tabular}{|l|c|c|c|c|c|c|c|c|}
\hline \multirow{2}{*}{ Centro } & \multicolumn{2}{|c|}{$\begin{array}{c}\text { Personales } \\
\text { generales }\end{array}$} & \multicolumn{2}{c|}{$\begin{array}{c}\text { Docentes } \\
\text { genéricas }\end{array}$} & \multicolumn{2}{c|}{$\begin{array}{c}\text { Específicas } \\
\text { EF }\end{array}$} & \multicolumn{2}{c|}{ Total } \\
\cline { 2 - 10 } & $M(\%)$ & $D E$ & $M(\%)$ & $D E$ & $M(\%)$ & $D E$ & $M(\%)$ & $D E$ \\
\hline Granada & $32,71(68,1)$ & 6,52 & $30,76(64,1)$ & 6,99 & $60,27(68,5)$ & 10,88 & $123,75(67,3)$ & 21,52 \\
\hline Valladolid & $32,29(67,3)$ & 6,24 & $28,83(60,1)$ & 7,63 & $59,12(67,2)$ & 9,17 & $120,24(65,3)$ & 20,27 \\
\hline La Laguna & $33,83(70,5)$ & 5,84 & $27,90(58,1)$ & 6,67 & $55,67(63,3)$ & 11,08 & $117,40(63,8)$ & 20,59 \\
\hline Valencia & $31,67(66,0)$ & 6,99 & $29,33(61,1)$ & 7,40 & $56,10(63,7)$ & 11,90 & $117,10(63,6)$ & 24,08 \\
\hline Santander & $29,83(62,1)$ & 8,34 & $28,27(58,9)$ & 9,51 & $53,59(60,9)$ & 13,92 & $111,68(60,7)$ & 29,25 \\
\hline Barcelona & $33,21(69,2)$ & 6,00 & $27,62(57,5)$ & 5,70 & $50,21(57,1)$ & 12,66 & $111,03(60,3)$ & 20,37 \\
\hline Albacete & $29,40(61,3)$ & 7,65 & $26,79(55,8)$ & 7,34 & $53,59(60,9)$ & 9,57 & $109,78(59,7)$ & 20,61 \\
\hline Murcia & $30,70(64,0)$ & 5,89 & $26,91(56,1)$ & 6,08 & $48,45(55,1)$ & 11,08 & $106,06(57,6)$ & 19,76 \\
\hline Madrid & $28,04(58,4)$ & 5,44 & $21,87(45,6)$ & 6,01 & $49,13(55,8)$ & 9,76 & $99,04(53,8)$ & 18,68 \\
\hline Sevilla & $26,22(54,6)$ & 6,50 & $22,65(47,2)$ & 6,93 & $48,09(54,6)$ & 10,19 & $96,96(52,7)$ & 19,91 \\
\hline
\end{tabular}

Nota: $\mathrm{EF}=$ Educación física. \% = Porcentaje respecto a la puntuación máxima. Puntuaciones máximas: competencias personales generales $=48$; competencias docentes genéricas $=48$; competencias específicas $\mathrm{EF}=88 ;$ total $=184$.

Fuente. Elaboración propia.

Existió una diferencia de hasta 7,61 puntos entre la media más alta y más baja de las universidades en lo que respecta a competencias personales generales $(15,8 \%$ sobre la puntuación máxima), 8,11 puntos en las competencias docentes (16,9\% sobre la puntuación máxima), 12,18 puntos con respecto a las competencias específicas de Educación Física (13,84\% sobre la puntuación máxima), y 26,79 puntos en relación al total de competencias (14,55\% sobre la puntuación máxima).

El análisis de la varianza determinó diferencias significativas entre centros en las tres dimensiones y en el total ( $p<0.001$ en todos los casos). Las pruebas post-hoc mostraron diversas diferencias entre grupos, que se muestran en la Tabla 3.

Como se puede apreciar en la dimensión de competencias personales generales, la universidad de Sevilla obtuvo puntuaciones significativamente inferiores al resto de universidades a excepción de la universidad de Madrid. Atendiendo a las competencias docentes genéricas se puede observar cómo tanto la universidad de Sevilla como la de Madrid obtuvieron una puntuación significativamente menor al resto de universidades. En la dimensión de competencias específicas de Educación Física, la universidad de Sevilla obtuvo resultados significativamente inferiores al resto de universidades, excepto con las universidades 
Estudios Pedagógicos XLIV, N $^{\circ}$ 2: 223-239, 2018

PERCEPCIONES DE ALUMNOS DEL GRADO EN MAESTRO EN EDUCACIÓN PRIMARIA CON MENCIÓN EN EDUCACIÓN FÍSICA SOBRE LA ADQUISICIÓN DE COMPETENCIAS

Tabla 3. Diferencias significativas entre centros en las dimensiones de competencias

\begin{tabular}{|c|c|c|c|c|c|c|c|c|c|c|}
\hline & & $\mathrm{Sev}$ & Mad & Mur & Albac & Barc & Sant & Valen & Laguna & Vallad \\
\hline \multirow[t]{9}{*}{$\mathrm{CPG}$} & Granada & $<, 001$ & ,007 & &, 031 & & & & & \\
\hline & Valladolid & $<, 001$ & & & & & & & & \\
\hline & La Laguna & $<, 001$ & ,013 & & & & & & & \\
\hline & Valencia & $<, 001$ & & & & & & & & \\
\hline & Santander & ,016 & & & & & & & & \\
\hline & Barcelona & $<, 001$ & & & & & & & & \\
\hline & Albacete &, 010 & & & & & & & & \\
\hline & Murcia & $<, 001$ & & & & & & & & \\
\hline & Madrid & & & & & & & & & \\
\hline \multirow[t]{9}{*}{ CDG } & Granada & $<, 001$ & $<, 001$ & ,041 & ,006 & & & & & \\
\hline & Valladolid & $<, 001$ & $<, 001$ & & & & & & & \\
\hline & La Laguna & ,012 &, 017 & & & & & & & \\
\hline & Valencia & $<, 001$ & $<, 001$ & & & & & & & \\
\hline & Santander & $<, 001$ & $<, 001$ & & & & & & & \\
\hline & Barcelona & ,029 & ,036 & & & & & & & \\
\hline & Albacete & $<, 001$ & ,006 & & & & & & & \\
\hline & Murcia & ,002 & ,013 & & & & & & & \\
\hline & Madrid & & & & & & & & & \\
\hline \multirow[t]{9}{*}{ CEEF } & Granada & $<, 001$ & $<, 001$ & $<, 001$ & ,001 & ,001 & ,009 & & & \\
\hline & Valladolid & $<, 001$ & ,001 & $<, 001$ & &, 034 & & & & \\
\hline & La Laguna &, 024 & & & & & & & & \\
\hline & Valencia & $<, 001$ & ,037 & ,002 & & & & & & \\
\hline & Santander & ,035 & & & & & & & & \\
\hline & Barcelona & & & & & & & & & \\
\hline & Albacete & ,004 & & & & & & & & \\
\hline & Murcia & & & & & & & & & \\
\hline & Madrid & & & & & & & & & \\
\hline \multirow[t]{9}{*}{ Total } & Granada & $<, 001$ & $<, 001$ & $<, 001$ & $<, 001$ & & ,032 & & & \\
\hline & Valladolid & $<, 001$ & $<, 001$ &, 042 & & & & & & \\
\hline & La Laguna & $<, 001$ &, 016 & & & & & & & \\
\hline & Valencia & $<, 001$ & ,001 & & & & & & & \\
\hline & Santander & $<, 001$ & & & & & & & & \\
\hline & Barcelona & & & & & & & & & \\
\hline & Albacete & $<, 001$ & & & & & & & & \\
\hline & Murcia & & & & & & & & & \\
\hline & Madrid & & & & & & & & & \\
\hline
\end{tabular}

Nota: $\mathrm{CPG}=$ Competencias personales generales $\mathrm{CDG}=$ Competencias docentes genéricas; $\mathrm{CEEF}=$ Competencias específicas de Educación Física; Total = Total de competencias .

Fuente. Elaboración propia. 
Estudios Pedagógicos XLIV, N $^{\circ}$ 2: 223-239, 2018

PERCEPCIONES DE ALUMNOS DEL GRADO EN MAESTRO EN EDUCACIÓN PRIMARIA CON MENCIÓN EN EDUCACIÓN FÍSICA SOBRE LA ADQUISICIÓN DE COMPETENCIAS

de Barcelona, Murcia y Madrid. Por último, tomando como referencia el total, la universidad de Granada obtuvo puntuaciones significativamente superiores a las universidades de Sevilla, Madrid, Murcia y Albacete. En el lado opuesto, la universidad de Sevilla obtuvo puntuaciones significativamente inferiores a siete de las diez universidades participantes.

\section{DISCUSIÓN}

Los resultados de este estudio mostraron que, a nivel general, los participantes perciben que sus estudios de grado han desarrollado sus competencias en torno a un $60 \%$ respecto a la máxima puntuación otorgada a la totalidad de las mismas, lo cual evidencia un amplio margen de mejora. También mostraron diferencias significativas entre las diversas competencias respecto a los centros de formación.

Las competencias que obtuvieron una mayor puntuación, entre "mucho" y "muchísimo", fueron la competencia genérica "Trabajo en equipo" y la competencia docente específica de Educación Física "Saber utilizar el juego como recurso didáctico y como contenido de enseñanza". Estos resultados son parcialmente coherentes con los obtenidos por la ANECA (2005b), tras encuestar a 180 profesores de estudios de magisterio de 18 universidades españolas a los que se le solicitó que valorasen la importancia de 23 competencias transversales (genéricas), 23 competencias comunes a todos los perfiles de maestro, y 18 competencias específicas de Educación Física. En este estudio, el "Trabajo en equipo" aparece en tercer lugar entre las competencias transversales, solo por detrás de "Capacidad de organización y planificación" y "Comunicación oral y escrita en la lengua materna", que en nuestro trabajo se sitúan entre el "medianamente" y "mucho". Romero et al. (2011) también encontraron, en su estudio con 439 estudiantes de magisterio en la especialidad de Educación Física de tres universidades andaluzas, que el trabajo en equipo fue la competencia transversal a la que otorgaron una mayor importancia. Sin embargo, en el estudio de Gutiérrez-García et al. (2011) la competencia "Trabajar en equipo con otros docentes" fue la tercera peor valorada por los 635 alumnos de las titulaciones de Maestro y Licenciado en Ciencias de la Actividad Física y del Deporte, solo por delante de "Participar en la gestión del centro" e "Informar e implicar a las familias". Desde nuestra experiencia, entendemos que el trabajo en equipo, entendido como competencia transversal a cualquier titulación universitaria, suele estar presente (incluso muy presente) en los estudios de magisterio, no solo por su valor intrínseco, sino también por pura "necesidad práctica": en unas aulas habitualmente masificadas (Valdés \& Gutiérrez, 2016; Viñao, 2013), una de las pocas opciones viables que tiene el profesorado universitario a la hora de encomendar y evaluar tareas de una cierta complejidad a los alumnos (e. g., elaboración de trabajos teóricos, portafolios, sesiones, unidades didácticas, etc.), es que dichas tareas se realicen de forma grupal. De este modo, y ya sea por convicción y/o necesidad del profesorado, los alumnos se encuentran frecuentemente trabajando en equipo, y de ahí su alta percepción de competencia al respecto. No obstante, esto no debe confundirse con la competencia específica "Trabajar en equipo con otros docentes", analizada en el trabajo de Gutiérrez-García et al. (2011). Lo que parece evidenciar esta disonancia, en nuestra opinión, es, simplemente, que el alumnado encuestado aún no se consideraba parte de esos "otros docentes".

Por lo que se refiere a "Saber utilizar el juego como recurso didáctico y como contenido de enseñanza", el estudio de la ANECA (2005b) lo sitúa como la segunda 
competencia específica más valorada por el profesorado universitario, solo por detrás de "Dominar la teoría y la didáctica específica de la Educación Física, los fundamentos y las técnicas de programación del área y diseño de las sesiones, así como las estrategias de intervención y de evaluación de los resultados". La utilización del juego como recurso y contenido también fue la competencia recibida más valorada en el estudio de Campos, Ries y Del Castillo (2011), realizado sobre 104 egresados de Magisterio de la especialidad de Educación Física de la Universidad de Sevilla, y también la competencia que dichos egresados valoraron como más utilizada en sus primeros trabajos. Por su parte, Romero et al. (2011) la situaron como la tercera competencia específica a la que los estudiantes otorgaron una mayor importancia, por detrás de "Capacidad de relación necesaria entre la actividad física con los hábitos de higiene, posturales y de salud, como contenido específico del área de EF y como contenido transversal junto con el resto de áreas que conforman el currículum" y "Promover hábitos y estilos de vida saludables, estableciendo relaciones transversales con todas las áreas del currículo". No obstante, es importante señalar que las valoraciones de los estudiantes en este último estudio fueron muy similares para todas las competencias más valoradas (transversales, básicas docentes y específicas de Educación Física), y que la pregunta de investigación se refería a la importancia percibida, y no a la percepción de competencia.

En el lado opuesto, cinco competencias obtuvieron en nuestro estudio una valoración de "poco/medianamente". Las dos menos valoradas, "Conocimiento de una lengua extranjera" y "Conocimientos de informática aplicados al ámbito de estudio", son posiblemente dos de las competencias que más han presionado al sistema educativo español durante los últimos años. Fiel reflejo de ello ha sido la creciente implantación de programas bilingües en los centros educativos (Hernando, Hortigüela \& Pérez-Pueyo, 2017a; Ramos \& Ruiz, 2011), así como de todo tipo de recursos informáticos orientados a facilitar el aprendizaje del alumnado (Moral \& Villalustre, 2010). De este modo, el crecimiento de programas bilingües y la implantación de las nuevas tecnologías en la educación -y a nivel social en general- probablemente han avanzado con más rapidez de lo que el sistema universitario y no universitario ha sido capaz (y/o ha estado dispuesto a enseñar) en las aulas.

Estos resultados son coherentes con los obtenidos en el estudio de la ANECA (2005b), en el que los conocimientos de informática relativos al ámbito de estudio y el conocimiento de una lengua extranjera figuraron entre las cinco competencias menos valoradas por el profesorado de estudios de magisterio. Particularmente para el perfil profesional de Maestro de Educación Primaria -Especialidad de Educación Física- estas competencias solo fueron puntuadas por encima de "Trabajo en un contexto internacional". Para los autores de este informe,

[...] no deja de sorprender que resulten tan poco valoradas dos competencias consideradas básicas hoy en día como son el conocimiento y uso de las nuevas tecnologías así como el de otro idioma. Asimismo, sorprende también que en un contexto tan europeizante como el que vivimos lo peor valorado de todo resulte ser precisamente el trabajo en un contexto internacional. (ANECA, 2005b, p. 85).

En este mismo sentido, Losada, Valverde y Correa (2012) valoraron como un retroceso la formación en tecnología educativa que reciben los actuales pedagogos, maestros y educadores sociales formados en las universidades públicas españolas en los nuevos grados adaptados al Espacio Europeo de Educación Superior, respecto a los que recibían 
Estudios Pedagógicos XLIV, N $^{\circ}$ 2: 223-239, 2018

PERCEPCIONES DE ALUMNOS DEL GRADO EN MAESTRO EN EDUCACIÓN PRIMARIA CON MENCIÓN EN EDUCACIÓN FÍSICA SOBRE LA ADQUISICIÓN DE COMPETENCIAS

los titulados que los precedieron. Para Cózar y Roblizo (2014, p. 121), "se ha eliminado buena parte de los planes de estudios la asignatura troncal 'Nuevas tecnologías aplicadas a la educación' [...] quedando la formación TIC relegada a un tratamiento transversal a criterio de los docentes universitarios".

Para el caso de las lenguas extranjeras, España se sitúa en el ámbito europeo en uno de los últimos lugares en cuanto a su dominio, y se ha considerado que, a pesar de los esfuerzos realizados, "las políticas educativas no consiguen aún impulsar de un modo efectivo el plurilingüismo" (Cuervo, Alonso \& Sabadell, 2013, p. 368). En estos esfuerzos, la Educación Física se ha valorado como "muy adecuada" (Salvador, ChivaBartoll \& Fazio, 2016, p. 121) o "una de las mejores" (Ramos \& Ruiz, 2011, p. 154) para integrar el aprendizaje de una lengua extranjera en el propio aprendizaje de los contenidos de la asignatura, siempre y cuando se utilicen metodologías de enseñanza basadas en la comunicación y en los aspectos fonológicos como el aprendizaje integrado de contenidos y lenguas extranjeras (AICLE; en inglés Content and language integrated learning, CLIL) (Hernando, Hortigüela \& Pérez-Pueyo, 2015; Hernando et al., 2017b; Hortigüela, Hernando y Pérez-Pueyo, 2016a). Se trata también de una asignatura que con frecuencia se incluye en los programas bilingües (Chiva-Bartoll, Isidori \& Fazio, 2016; Hernando, et al., 2017a), y de hecho se ha convertido en una competencia muy valorada -incluso exigidaen la contratación de profesorado en centros privados y concertados o para el acceso a centros públicos en los que la Educación Física se imparte en inglés. Como apuntan Coral y Lleixá (2013, p. 79), “En la actualidad el conocimiento funcional de la lengua inglesa es un factor clave que condiciona el futuro socio-laboral de los estudiantes y cuya carencia incrementa la desigualdad de oportunidades". En síntesis, el alumnado participante en nuestro estudio parece ser muy consciente de las demandas sociales existentes respecto a estas dos competencias, y reclamar una mejora en la formación que recibe al respecto.

Por otra parte, las competencias "Participar en la gestión del Centro", "Elaborar y poner en práctica programas de Educación Física que faciliten la inclusión efectiva de los alumnos con necesidades educativas especiales", e "Informar e implicar a las familias" también estuvieron entre las menos valoradas. En relación con la segunda de ellas, también puede apuntarse que la competencia docente general "Elaborar y poner en práctica estrategias de atención a la diversidad" obtuvo una valoración ligeramente por encima del "medianamente", con una puntuación media de 2,18. Estos resultados están en la misma línea que los obtenidos en los estudios de Camacho y Padrón (2006), realizado sobre una muestra de 192 diplomados y licenciados futuros docentes, y Gutiérrez-García et al. (2011), quienes contaron para su estudio con las opiniones de 72 profesores y 635 alumnos de las titulaciones de Maestro y Licenciado en Ciencias de la Actividad Física y del Deporte. El ya citado estudio de la ANECA (2005b, p. 91), mostró resultados más difíciles de interpretar, situando, por un lado, las competencias "Capacidad para promover la calidad de los contextos (aula y centro) en los que se desarrolla el proceso educativo, de modo que se garantice el bienestar de los alumnos" y "Capacidad para colaborar con los distintos sectores de la comunidad educativa y del entorno" entre las menos valoradas por los profesores universitarios de magisterio, mientras que entre las más valoradas figuraron otras relacionadas con las que aquí se discuten como "Respeto a las diferencias culturales y personales de los alumnos y demás miembros de la comunidad educativa" o "Capacidad para desempeñar la función tutorial, orientando a alumnos y padres y coordinando la acción educativa referida a su grupo de alumnos". En cualquier caso, el propio documento 
de la ANECA (2005b) planteaba tres ejes de actuación respecto a la formación de maestros, desarrollados en ocho objetivos, entre los que se encuentra la capacidad para relacionarse con otros agentes sociales del entorno educativo, así como la capacidad de trabajo en equipo en el centro educativo. También señala la "Acentuación del carácter práctico de los estudios de magisterio y la relación teoría-práctica" (p. 194), que bien puede interpretarse, al menos parcialmente, como la necesidad que van a tener los futuros maestros de saber enfrentarse a la realidad de las aulas en las que prima la diversidad. Una década después de que comenzasen a elaborarse los títulos de grado de magisterio, la mejora en estas competencias sigue pendiente, al menos desde la perspectiva del alumnado.

En la comparación entre centros de formación de profesorado, aspecto que apenas ha sido tratado en la literatura (aunque con objetivos diferentes, véase por ejemplo Pérez et al., 2012), nuestro estudio ha detectado diferencias significativas en la percepción de competencia del alumnado de diversas universidades, aunque en términos generales estas diferencias están bastante localizadas y la mayoría de centros obtiene puntuaciones similares. Han existido, en este sentido, numerosos factores tendentes a la homogeneización de las enseñanzas de magisterio, tanto a nivel europeo como específicamente en España (véase Tiana, 2013). Sirva, a modo de ejemplo, la propia normativa reguladora del título (Orden ECI/3857/2007, de 27 de diciembre, por la que se establecen los requisitos para la verificación de los títulos universitarios oficiales que habiliten para el ejercicio de la profesión de Maestro en Educación Primaria), así como documentos no normativos, pero respaldados a nivel oficial, como fue el caso del Libro Blanco del título de grado en magisterio (ANECA, 2005b). Asimismo, tampoco pueden perderse de vista las resistencias al cambio características de la docencia universitaria. Según se expresaba en el propio Libro Blanco,

[...] en definitiva, quizás lo más destacado de todo resulte la visión más bien anclada en el rol convencional del maestro: alguien que se expresa bien en su lengua, que organiza y planifica su trabajo y que, esto sí, reconoce la multiculturalidad; alguien, al mismo tiempo, para el que una lengua extranjera (excepto para los de ese perfil) y las nuevas tecnologías suponen una formación necesaria pero poco valorada frente a otras competencias; y alguien para quien la mayor parte de las propuestas de innovación educativa recogidas por la literatura de los últimos años permanecen al fin en un segundo plano. La prevalencia de esta perspectiva tradicional predominante ha supuesto una sorpresa, en la medida que las expectativas de los miembros del grupo de trabajo se enmarcaban en la dirección contraria, y también un motivo de preocupación de cara a la resistencia al cambio del modelo formativo que se propone desde estas páginas.

Por el contrario, también existen factores que pueden explicar las diferencias entre los centros de formación del profesorado. Tiana (2013, p. 52) señala que la actual normativa reguladora de la formación inicial del profesorado "permite un considerable grado de flexibilidad y de capacidad de actuación por parte de las universidades", e igualmente las diversas normativas autonómicas pueden crear diferentes marcos de desarrollo en los centros universitarios. Para este autor, que considera "incompleta" la reforma del actual modelo de formación inicial del profesorado, existen aspectos criticables como la interacción con el sistema educativo o la mejora de la orientación práctica de los estudios. Estos y otros aspectos, prioridades, líneas de actuación, etc., referidos abundantemente 
en la literatura (ANECA, 2005b; Esteve, 2006; Valdés \& Gutiérrez, 2016), dependen en gran medida del profesorado de los diversos centros y de su capacidad para trabajar de forma coordinada para proporcionar al alumnado experiencias auténticas de aprendizaje que promuevan un elevado grado de adquisición de competencias. La evidencia disponible sugiere que la calidad de los profesores es el principal factor condicionante del aprendizaje de los estudiantes en la escuela. Así, las realidades específicas de los diversos centros de formación parecen estar dando como resultado que sus egresados tengan diferentes percepciones de competencia al finalizar sus estudios.

\section{CONCLUSIONES}

Los alumnos del Grado en Maestro de Educación Primaria con mención en Educación Física participantes en este estudio consideraron que su formación inicial como maestros había logrado desarrollar sus competencias personales generales, docentes genéricas y específicas de Educación Física de forma similar, en un punto intermedio entre "medianamente" y "mucho". Las competencias relacionadas con el trabajo en equipo y con la utilización del juego como recurso didáctico fueron las mejor valoradas, por encima de "mucho", mientras que aquellas relacionadas con el conocimiento de una lengua extranjera, conocimientos de informática relativos al ámbito de estudio, participación en la gestión del centro, elaboración y puesta en práctica programas de Educación Física inclusivos, e informar e implicar a las familias fueron valoradas por debajo de "medianamente". Ninguna competencia fue valorada por debajo de "poco". Estos resultados, que actualizan y están en línea con los expresados en otras investigaciones, invitan a seguir reflexionando sobre el trabajo que se desarrolla en los centros de formación del profesorado y sobre cómo éste podría reorientarse para mejorar la percepción de adquisición de competencias del alumnado.

Esta investigación también concluye que existen similitudes y diferencias de percepción atendiendo al centro de realización de los estudios. Esto se explica a partir de las tensiones existentes entre la homogeneización y diversificación de los centros formativos, en los que se destaca al profesorado como pieza clave. Como futuras líneas de investigación planteamos la ampliación de la muestra utilizada a un mayor número de universidades públicas y también a las universidades privadas con las que se podrían realizar estudios comparativos. Ello permitiría realizar un seguimiento sobre la percepción de competencias del alumnado, así como proponer estudios más detallados de aquellos centros que obtengan mejores resultados. Obviamente, esta valoración no debería basarse únicamente en la percepción del alumnado, sino tener en cuenta las opiniones de otros colectivos, como profesorado, egresados, empleadores y las evaluaciones implementadas a nivel institucional. Todos estos aspectos constituyen igualmente líneas de investigación orientadas a la evaluación de la formación inicial del profesorado de Educación Física.

\section{REFERENCIAS BIBLIOGRÁFICAS}

ANECA. (2005a). Libro Blanco. Título de Grado en Ciencias de la Actividad Física y el Deporte. Madrid: Agencia Nacional de Evaluación de la Calidad y la Acreditación. 
ANECA. (2005b). Libro Blanco. Título de Grado en Magisterio (Vol. 1). Madrid: Agencia Nacional de Evaluación de la Calidad y la Acreditación.

Brown, S., \& Pickford, R. (2013). Evaluación de habilidades y competencias en Educación Superior. Madrid: Narcea.

Camacho, H. M., \& Padrón, M. (2006). Malestar docente y formación inicial del profesorado: percepciones del alumnado. Revista interuniversitaria de formación del profesorado, 20(2), 209-230. Recuperado de http://www.aufop.com/aufop/uploaded_files/articulos/1209214983. pdf

Campos, M. C., Ries, F., \& Del Castillo, O. (2011). Análisis de las competencias adquiridas y utilizadas por los egresados maestros en Educación Física. Revista Internacional de Ciencias del Deporte, 24(7), 216-229. Recuperado de http://www.cafyd.com/REVISTA/02405.pdf

Cañabate, D., Aymerich, M., Falgàs, M., \& Gras, M. E. (2014). Metodologías docentes: motivación y aprendizaje percibidos por los estudiantes universitarios. Educar, 50(2), 427-441.

Carreras, J., \& Perrenoud, P. (2005). El debat sobre les competències a l'ensenyament universitari. Barcelona: Universitat de Barcelona.

Coral, J., \& Lleixá, T. (2013). Las tareas en el aprendizaje integrado de educación física y lengua extranjera (AICLE). Determinación de las características de las tareas mediante el análisis del diario de clase. Retos: nuevas tendencias en educación física, deporte y recreación, 24, 79-84. Recuperado de https://dialnet.unirioja.es/descarga/articulo/4473846.pdf

Cózar, R., \& Roblizo, M. J. (2014). La competencia digital en la formación de los futuros maestros: percepciones de los alumnos de los Grados de Maestro de la Facultad de Educación de Albacete. RELATEC: Revista Latinoamericana de Tecnología Educativa, 13(2), 119-133. doi:10.17398/1695-288X.13.2.119

Cuervo, A. E., Alonso, R. M., \& Sabadell, M. (2013). Reflexiones en torno a la lengua francesa en Castilla y León: secciones bilingües y formación de los profesores de disciplinas no lingüísticas (DNL). REDU. Revista de Docencia Universitaria, 11(1), 365-388. doi:10.4995/redu.2013.5605

Chiva-Bartoll, O., Isidori, E., \& Fazio, A. (2016). Educación Física bilingüe y pedagogía crítica: una aplicación basada en el Judo. Retos: nuevas tendencias en educación física, deporte y recreación, 28, 110-115. Recuperado de https://recyt.fecyt.es/index.php/retos/article/view/34812/19075

DeVellis, R. F. (2003). Scale Development: Theory and applications (2nd ed.). Thousand Oaks, CA: Sage.

Díaz, J., \& Hervella, F. R. (Eds.). (2017). Informe equidad educativa. La sombra de la inversión educativa en España. En busca de la gratuidad y la equidad en la educación. España: Educo y Ayuda en Acción.

Díez, M. C., Pacheco, D. I., García, J. N., Martínez, B., Robledo, P., Álvarez, M. L., . . Carbonero, M.A., (2009). Percepción de los estudiantes universitarios de educación respecto al uso de metodologías docentes y el desarrollo de competencias ante la adaptación al EEES: datos de la Universidad de Valladolid. Aula abierta, 37(1), 45-56.

Esteve, J. M. (2006). La profesión docente en Europa: perfil, tendencias y problemática. La formación inicial. Revista de Educación, 340, 19-40. Recuperado de http://www.revistaeducacion.mec.es/ re340/re340_03.pdf

Gabarda, V., Rodríguez, A., Rodríguez, M. D., \& Cantón, I. (2017). La competencia digital en estudiantes de magisterio. Análisis competencial y percepción personal del futuro maestro. Educatio siglo XXI: Revista de la Facultad de Educación, 35(2), 253-274.

Galvis, R.M.(2007). De un perfil docente tradicional a un perfil docente basado en competencias. Acción Pedagógica, 16(1), 48-57. Recuperado de http://www.saber.ula.ve/handle/123456789/17284

Gallego, J. L., \& Rodríguez, A. (2014). Percepción del alumnado universitario de Educación Física sobre su competencia comunicativa. Movimento: revista da Escola de Educação Física, 20(2), 425-444.

Gallego, J. L., \& Rodríguez, A. (2015). Competencias comunicativas de maestros en formación de 
Estudios Pedagógicos XLIV, N $^{\circ}$ 2: 223-239, 2018

PERCEPCIONES DE ALUMNOS DEL GRADO EN MAESTRO EN EDUCACIÓN PRIMARIA CON MENCIÓN EN

EDUCACIÓN FÍSICA SOBRE LA ADQUISICIÓN DE COMPETENCIAS

educación especial. Educación y educadores, 18(2), 209-225. doi:10.5294/edu.2015.18.2.2

Gutiérrez-García, C., Pérez-Pueyo, Á., Pérez-Gutiérrez, M., \& Palacios-Picos, A. (2011). Percepciones de profesores y alumnos sobre la enseñanza, evaluación y desarrollo de competencias en estudios universitarios de formación de profesorado. Cultura y Educación, 23(4), 499-514.

Hernando, A., Hortigüela, D., \& Pérez-Pueyo, A. (2015). Análisis de la implantación de la educación física bilingüe en Castilla y León. In M. A. Iglesias (Ed.), II Jornada de Doctorandos de la Universidad de Burgos (pp. 33-37). Burgos: Universidad de Burgos.

Hernando, A., Hortigüela, D., \& Pérez-Pueyo, Á. (2017). Percepción de coordinadores de programas bilingües y docentes de Educación Física en inglés en secundaria sobre el proceso de implantación y desarrollo del bilingüismo en la Comunidad de Castilla y León. Retos: nuevas tendencias en educación física, deporte y recreación, 33, 63-68. Recuperado de https://recyt.fecyt.es/index. $\mathrm{php} / \mathrm{retos} / \mathrm{article} / \mathrm{view} / 54423$

Hernando, A., Hortigüela, D., Pérez-Pueyo, A., \& Fernández-Río, J. (2017). La Educación Física en secciones bilingües de inglés: teoría y práctica. Buenos Aires: Miñó y Dávila.

Hortigüela, D., Hernando, A., \& Pérez-Pueyo, A. (2016). ¿Qué estrategias metodológicas utiliza el profesor de Educación Física bilingüe en el aula? In M. I. Amor, J. L. Luengo-Almena \& M. Martínez (Eds.), Educación intercultural: metodología de aprendizaje en contextos bilingües (pp. 103-106). Granada: Atrio.

Hortigüela, D., Pérez-Pueyo, Á., \& Fernández-Río, J. (2016). Influencia de las experiencias vivenciadas por el alumnado en el desempeño de futuras competencias docentes. Contextos educativos: Revista de educación, 19, 25-41. doi:10.18172/con.2742

Iglesias, M. J. (2009). Elaboración y validación de un instrumento diagnóstico para la percepción de las competencias emocionales en estudiantes universitarios de Educación. Revista Española de Orientación y Psicopedagogía, 20(3), 300-311.

Izuzquiza, D., Echeita, G., \& Simón, C. (2015). La percepción de estudiantes egresados de magisterio en la Universidad Autónoma de Madrid sobre su competencia profesional para ser 'profesorado inclusivo': un estudio preliminar. Tendencias pedagógicas, 26, 197-216.

López-Pastor, V. M., \& Pérez-Pueyo, Á. (Eds.). (2017). Evaluación formativa y compartida en educación: experiencias de éxito en todas las etapas educativas. León: Universidad de León.

Lorente-Catalán, E., Montilla, M. J., Joven, A., Graells, A., \& Castel, D. (2017). ¿Perciben del mismo modo alumnado y profesorado el desarrollo de competencias docentes? El caso de un grado de ciencias de la actividad física y el deporte. Infancia, Educación y Aprendizaje, 3(2), 588-593.

Losada, D., Valverde, J., \& Correa, J. M. (2012). La tecnología educativa en la Universidad Pública Española. Píxel-Bit, vol. 41, 133-148. doi:10.12795/pixelbit

Lozano, R. (2011). Las 'TIC/TAC': de las tecnologías de la información y comunicación a las tecnologías del aprendizaje y del conocimiento. Anuario Think EPI, 5, 45-47.

Martínez, J. B. (2016). Crisis Económica y Financiación Educativa: Evolución de la Inversión (2009-2013). Madrid: Federación Enseñanza CC.OO.

Montero, I., \& León, O. G. (2007). A guide for naming research studies in Psychology. International journal of clinical and health psychology, 7(3), 847-862. Recuperado de http://www.aepc.es/ ijchp/GNEIP07_es.pdf

Moral, M. E. D., \& Villalustre, L. (2010). Formación del profesor 2.0: desarrollo de competencias tecnológicas para la escuela 2.0. Magister: Revista miscelánea de investigación, 23, 59-69. Recuperado de https://dialnet.unirioja.es/descarga/articulo/3403432.pdf

Parellada, M. (Ed.). (2015). Informe CYD 2015. La contribución de las universidades españolas al desarrollo. Barcelona: Fundación Conocimiento y Desarrollo.

Pérez, L. A., López, M. C., Couto, J. M., \& Navarro, J. G. (2012). ¿Depende la satisfacción estudiantil de la calidad real de la facultad o de la percepción individual de ésta? Análisis comparativo entre facultades privadas y públicas. ReiDoCrea: Revista electrónica de investigación y docencia creativa, 1, 29-37. 
Perrenoud, P. (2004). Diez competencias para enseñar. Barcelona: Graó.

Pozo, C., \& Bretones, B. (2015). Dificultades y retos en la implantación de los títulos de grado en las universidades españolas. Revista de Educación, 367, 147-172. doi:10.4438/1988-592XRE-2015-367-286

Ramos, F., \& Ruiz, J. V. (2011). La educación física en centros bilingües de primaria inglés-español: De las singularidades propias del área a la elaboración de propuestas didácticas prácticas con AIBLE. Revista española de lingüística aplicada, 24, 153-170.

Romero-Martín, R., Castejón-Oliva, F.-J., López-Pastor, V.-M., \& Fraile-Aranda, A. (2017). Evaluación formativa, competencias comunicativas y TIC en la formación del profesorado. Comunicar: Revista científica iberoamericana de comunicación y educación, 52, 73-82. doi:10.3916/C52-2017-07

Romero, C., Zagalaz, M. L., Romero, M. N., \& Martínez, E. J. (2011). Importancia de las competencias profesionales de los Maestros en Educación Física expresadas por los estudiantes. Retos: nuevas tendencias en educación física, deporte y recreación, 19, 62-68. Recuperado de http://recyt. fecyt.es/index.php/retos/article/view/34641

Romero, M., \& Oliveira, J. M. d. (2011). La generación net se tambalea: percepción del dominio de las TIC de estudiantes de magisterio. Education in the knowledge society (EKS), 12(3), 265-283.

Romero, M. d. R., Castejón, F. J., López-Pastor, V. M., \& Fraile, A. (2017). Evaluación formativa, competencias comunicativas y TIC en la formación del profesorado. Comunicar: Revista científica iberoamericana de comunicación y educación, 52, 73-82. doi:10.3916/C52-2017-07

Salvador, C., Chiva-Bartoll, O., \& Fazio, A. (2016). Características del aprendizaje integrado de contenidos de educación física y lengua extranjera. Retos: nuevas tendencias en educación física, deporte y recreación, 29, 120-125. Recuperado de https://dialnet.unirioja.es/descarga/ articulo/5400856.pdf

Sierra, R. (1994). Técnicas de Investigación social. Madrid: Paraninfo.

Tiana, A. (2013). Los cambios recientes en la formación inicial del profesorado en España : una reforma incompleta. Revista Española de Educación Comparada, 22, 39-58. doi:10.5944/ reec.22.2013.9322

Valdés, V., \& Gutiérrez, P. (2016). Desafíos en la formación inicial del profesorado. Un análisis desde las voces de sus protagonistas. Revista Internacional de Formação de Professores, 1(3), 45-58. Recuperado de https://itp.ifsp.edu.br/ojs/index.php/RIFP/article/view/400/327

Vallejo, M., \& Molina, J. (2014). La evaluación auténtica de los procesos educativos. Revista Iberoamericana de educación, 64, 11-25.

Viaña, D. (2017). Presupuestos 2018. El Gobierno volverá a reducir el gasto en Sanidad, Educación y Protección Social el próximo año. El Mundo. Retrieved from http://www.elmundo.es/economia/ macroeconomia/2017/10/30/59f0dcc2468aeb89168b45de.html

Viñao, A. (2013). Modelos de formación inicial del profesorado de educación secundaria en España: siglos XIX-XXI. Revista Española de Educación Comparada, 22, 19-37. doi:10.5944/ reec.22.2013.9321 
